\title{
NEW MEASUREMENT METHOD FOR VERY THIN SAMPLES WITH VERY HIGH ACCURACY CONTROL OF THE LEVEL OF THE EMISSIVE POWER IN MICROWAVE ABSORPTION (8.5-9.5 GHz)
}

\author{
Constantin HUŢANU \\ "1 Decembrie 1918" University of Alba Iulia, Faculty of Sciences, Romania \\ e-mail: hutanu_constantin@yahoo.co.uk
}

\begin{abstract}
Dielectric materials, especially ferroelectrics, have increasingly specialized applications in the manufacture of IT industry components. At the same time, the working frequency of the microprocessors increases steadily, reaching the microwave domain. This situation requires a very thorough study on the behaviour of dielectric materials at ultra-high frequencies and with very precise determination of their electrical and magnetic properties at these frequencies. A very important aspect in such a study is the evaluation with an extremely high accuracy, the degree of absorption of these materials in the microwave domain. The creation of an experimental installation dedicated to the very precise testing of these dielectrics at microwave frequencies, is a special challenge in the area of scientific research. The content of this article describes very accurately and completely such an experimental installation, as well as how to ensure a very high accuracy of the measurement accuracy. The samples of dielectric materials used in these measurements are made of very thin films.
\end{abstract}

KEYWORDS: high accuracy, microwave diagnosis, ferroelectrics, absorption

\section{Introduction}

Considering the use of dielectric materials in more and more applications in the manufacture of electronic components, a detailed study is required regarding their behaviour at increasing frequencies. In the electronic circuit manufacturing industry for the IT domain, the working frequency of these electronic components has increased progressively year by year. Currently, the working frequency of the microprocessors and memories that equip the computing systems, as well as other devices used in the process of testing and controlling electronic products and production flows, has reached the microwave value field. The electromagnetic field with microwave frequencies, radiated by these components, can influence or disrupt the functioning of other electronic components in the immediate vicinity of the radiative components, by inducing parasitic electric currents with a frequency equal to that of the radiated microwave field or with frequencies corresponding to higher order harmonics. This undesirable situation can be avoided using three methods, namely: a) using highly absorbent polymeric materials for the electromagnetic field of microwaves, in the manufacture of radiative electronic component body/capsule;

b) using, for electronic components at risk of being disturbed by the radiative microwave field, materials immune to microwave frequencies.

c) using highly absorbent materials for the electromagnetic field of microwave irradiated, in the manufacture of capsules of electronic components at risk of disturbed or abnormal operation when they would be under the influence of an electromagnetic field of microwaves.

In most cases, the second option is incompatible with the process of manufacturing electronic or extremely expensive components.

Evaluating the first and last variant, we can easily see that for the manufacture of capsules of radiative electronic components, as well as for the manufacture of capsules of electronic components at risk of functioning in the presence of an electromagnetic field of microwaves, the use of highly absorbent dielectric materials is required.

Electronic test stands, in the field of microwave frequencies, for the study of the behaviour of solid 
dielectric materials, in most cases, they involve using Gunn diodes to generate the electromagnetic field of microwaves. The diode is based on the so-called Gunn effect. J. B. Gunn discovered in 1963 that when a constant, relatively high electrical voltage was applied to an n-type gallium arsenide crystal (GaAs semiconductor), it could cause rapid, static current fluctuations. It was demonstrated that in crystals of very short length these current fluctuations translated into continuous oscillations, the frequencies of which are determined by the crystal's length and lie in the microwave range. The Gunn effect is based on the fact that when energy is supplied to n-type semiconductors with unevenly doped regions electrons it can jump from one energy band into a higher energy band. In the higher energy bands, the electrons are less mobile, which is noticeable in that greater field strength arises at a lower current. This means that for the Gunn effect a lower voltage is needed at a higher current and a lower current at a higher voltage. The dynamic resistance is thus negative in this range (Fig. 1).

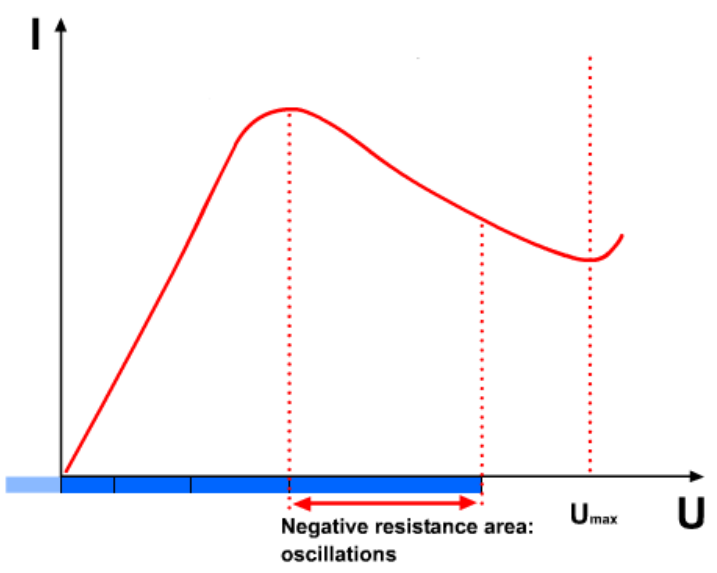

Fig. 1. Current-voltage characteristic of the Gunn diode

Using an assembly Gunn diode - adjustable resonant cavity [1], we can get it a variable frequency electromagnetic field generator, in the microwave domain.

One of the disadvantages of this type of microwave generator is that the power of the microwave signal is not constant across the frequency range. Using the absorption method in the study of the absorbing properties of solid-state dielectrics [2, 3] an unknown level of microwave signal for each frequency generated can be interpreted as an absorption phenomenon. This situation can lead to false conclusions regarding the absorbing properties of the studied dielectric $[4,5]$.

The experimental installation that will be described in the next chapter eliminates this risk, having as its main characteristic a control of the stability of the microwave signal level, with an ultrahigh accuracy.

\section{Theoretical sample model}

A transmission line can be represented by the circuit diagram shown below. The line is fed with the harmonic voltage $U_{o} e^{j \omega t}$. The source has the internal resistance $\mathrm{Z}_{\mathrm{i}}$. The transmission line of the length 1 is loaded at one end with the load resistance $\mathrm{Z}_{\mathrm{L}}$ (Fig. 2).

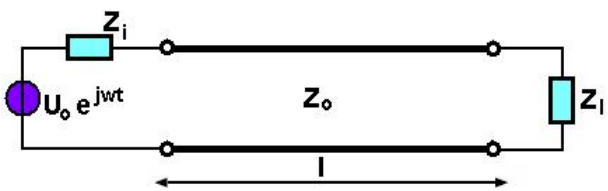

Fig. 2. General transmission line with source resistance $Z_{i}$ and load resistance $Z_{l}$

The basic voltage and current distribution can be computed using the wave equations:

$$
\underline{U}(z)=U_{0}^{+} e^{-\gamma z}+U_{0}^{-} e^{\gamma z} ; \underline{I}(z)=I_{0}^{+} e^{-\gamma z}+I_{0}^{-} e^{\gamma z}
$$

Where $\mathrm{U}_{0}^{+}$and $\mathrm{U}_{0}^{-}$respectively $\mathrm{I}_{0}^{+}$and $\mathrm{I}_{0}^{-}$are random complex constants. The abbreviation $\gamma$ designates the so-called propagation constant. It has the form:

$$
\gamma=\sqrt{\left(R^{\prime}+j \omega L^{\prime}\right)\left(G^{\prime}+j \omega C^{\prime}\right)}=\alpha+j \beta
$$

whereby R', L', G', C' are quantities of unit length.

The real component $\alpha$ is called the attenuation constant and the imaginary component $\beta$ is called the phase constant. There is a direct relationship existing between current and voltage on the transmission line, which depends on the characteristic impedance of the waveguide segment.

A uniform transmission line is defined as the one whose dimensions and electrical properties are identical at all planes transverse to the direction of propagation.

In a uniform transmission line, the dielectric sample may be modelled by the following circuit representation:

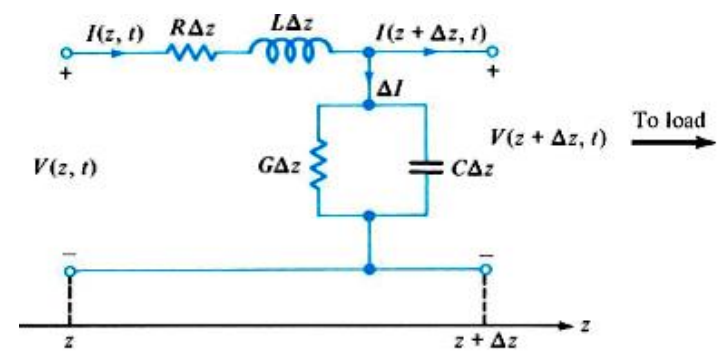

Fig. 3. The dielectric sample in TEM model 
Since the thickness of the studied dielectric material $\Delta z$ can always be chosen small compared to the operating wavelength, an individual section of line may be analyzed using ordinary ac circuit theory. In the following analysis, we let $\Delta \mathrm{z} \rightarrow 0$, so the results are valid at all frequencies (hence for any physical time variation).

It is possible that at a certain frequency of the microwave field the resonance condition [6] will be satisfied and consequently the energy absorbed from the microwave field that crosses the dielectric sample will be maximum. This situation can be highlighted by the measuring probe placed at the end of the waveguide, which will measure an electrical voltage diminished in proportion to the degree of absorption of the electromagnetic incident energy after crossing the dielectric sample $[7,8]$.

\section{Measurement bench}

The measuring stand is made up of modular microwave components, manufactured by LucasNülle $\mathrm{GmbH}$, and is intended for academic education and research. The measurement systems can be configured in two experimental variants:

a) according to the principle schemes made by the production company, aiming at the study of certain characteristics, phenomena and particularities specific to microwave technologies;

b) according to the user's conception, aiming at a certain experimental purpose.

Microwaves are generated by a Gunn oscillator and their propagation in conjunction with rectangular waveguides.

The recommended frequency band for experimental measurements using various configurations of these components is 8.5 to $9.6 \mathrm{GHz}$. Within the limits of this frequency band, all the components that make up the experimental installation keep approximately constant their catalogue features, the variations are less than $0.5 \%$.

The experimental installation consists of: Gunn oscillator, isolator, variable attenuator, slotted line, coaxial measuring probe for slotted guide, $\mathrm{N}$ socket/SMA plug adapter, an LNC (Low Noise Converter), SMA cable, waveguide adapter, R100/N adapter and still an LNC with an SMA cable and another X-Band Measurement Interface connected by another Unitrain-I Experimenter. The experimental installation is actually an embed measurement system, because the hard part is connected to a dedicated data acquisition system/X-Band Measurement Interface that sends the signals in digital format to a software package installed on a PC. The software package takes over processes the data and interprets the results obtained after processing.
Both operations control the block and the measuring instruments are virtual.

This virtual instrument is used to adjust the power to the Gunn oscillator and simultaneously operates as a display instrument for the microwave measuring receiver.

The experimental card/X-Band Measurement Interface is connected to a hard interface (Unitrain-I Interface and Unitrain-I Experimenter) that communicates with the PC through a USB cable.

The frequency of the oscillator that generates the microwave signal can be changed by rotating the micrometric screw that actuates a piston inside the resonant cavity. The illustration below (Fig. 4) is a schematic depiction of the structure of simple, mechanically tuned Gunn oscillator used in waveguide technology. Under the carriage the tube is blocked or shorted off to create a resonant cavity. The distance to this should be selected so that the load on the Gunn diode forms a parallel resonant circuit, which occurs at a distance corresponding to about $\lambda / 4$. In combination with the entry hole, and the frequency calibrating screw, the output can be transformed to match the characteristic impedance of the waveguide.

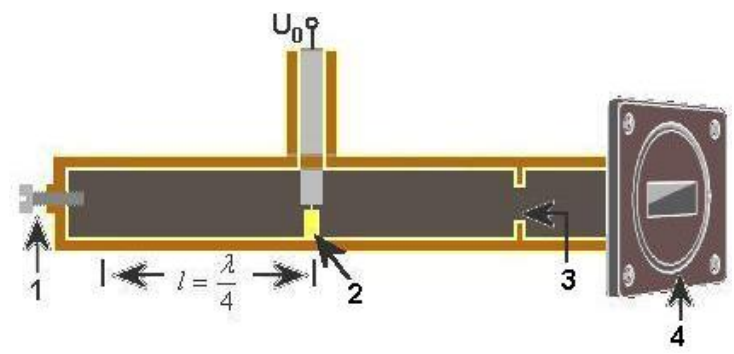

Fig. 4. Schematic design of a mechanically tunable Gunn oscillator

where: 1 Micrometer screw for frequency calibration, 2 Gunn diode, 3 Entry hole, 4 Flange.

The maximum power generated by the Gunn oscillator is $+17 \mathrm{~dB}(50 \mathrm{~mW})$ and the frequency band is between 8.5 to $9.9 \mathrm{GHz}$.

The isolator is a non-reciprocal waveguide element and is primarily used for decoupling the oscillator from waves coming back from the waveguide assembly further down the line.

An LNC (also referred to as a low-noise signal converter or input amplifier) is used to convert high frequencies into lower frequencies (IF). The X-band LNC in conjunction with an internal oscillator of 10 $\mathrm{GHz}$ and mixer receives the X-band signal to be measured with a frequency around $9 \mathrm{GHz}$ and generates a proportional signal with an intermediate frequency (IF) in the range of $1 \mathrm{GHz}$. This IF signal is supplied by the LNC to the receiver card of the UniTrain-I Experimenter via an SMA cable and there 
it is further processed and rectified. The LNC is also supplied with operating voltage via the SMA cable.

The attenuator can change the level of the microwave signal, taken from the output of the isolator, between the limits 0 to $-20 \mathrm{~dB}$. The value of the attenuation level is adjusted by rotating a micrometric screw. In order to be able to increase or decrease the microwave signal level at the output of the variable attenuator, depending on the direction of variation of the signal power at different frequencies at the output of the Gunn oscillator, the micrometric screw of the variable attenuator is positioned at division 2. This position of the micrometric screw corresponds to an attenuation of $-10 \mathrm{~dB}$ introduced additionally by the variable attenuator. The increase or decrease of the additional attenuation level with an amount between 0 and $10 \mathrm{~dB}$ compared to the $-10 \mathrm{~dB}$ attenuation set by positioning the micrometric screw at division 2, compensates for the small power variations of the signal generated by the Gunn oscillator when the generated frequency is changed.

The experimental installation of measurement can be seen in the following image (Fig. 5).

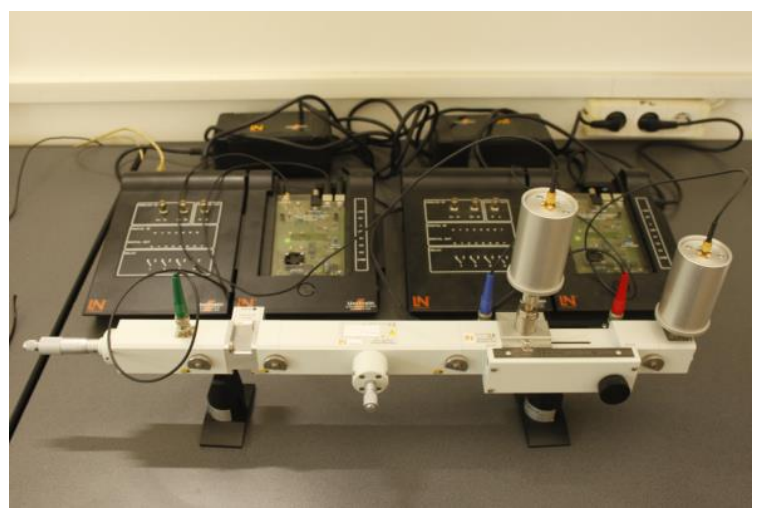

Fig. 5. The experimental installation of measurement

The operating mode is as follows:

a) Set the micrometer screw on the Variable Attenuator to a mark of " $\| "$ with number 0 at - symbol;

b) Set the switch to the ON position on both UniTrain-I Experiments;

c) Wait until both LEDs light up green;

d) Open the L@Bsoft application on both PCs;

e) Open the Microwave Control Center from the Instruments menu;

f) Set the voltage of the voltage for polarization of the Gunn diode to $8.22 \mathrm{~V}$;

g) Rotate the micrometric screw of the Gunn oscillator until you get the value of $8.60 \mathrm{GHz}$ in the FREQUENCY $(\mathrm{GHz})$ window on the virtual instrument MiCROWAVE CONTROL CENTER; h) Read and note the value of the power of the microwave signal generated by the Gunn oscillator, measured at the end of the waveguide and displayed in the LEVEL window of MICROWAVE CONTROL CENTER;

i) Repeat operations from items g) and $h$ ) for frequencies of $8.70 \mathrm{GHz}, 8.80 \mathrm{GHZ}, \ldots 9.50 \mathrm{GHz}$;

The variations in the power level of the microwave signal generated by the Gunn oscillator when the working frequency is changed can be seen in Fig. 6. Bringing this level to a constant value (arbitrarily chosen from the set of previously obtained values), for any frequency in the band $8.60-9.50 \mathrm{GHz}$, is done by turning to the right or to the left (as the case may be) the micrometric screw of the Variable Attenuator (Fig. 7).

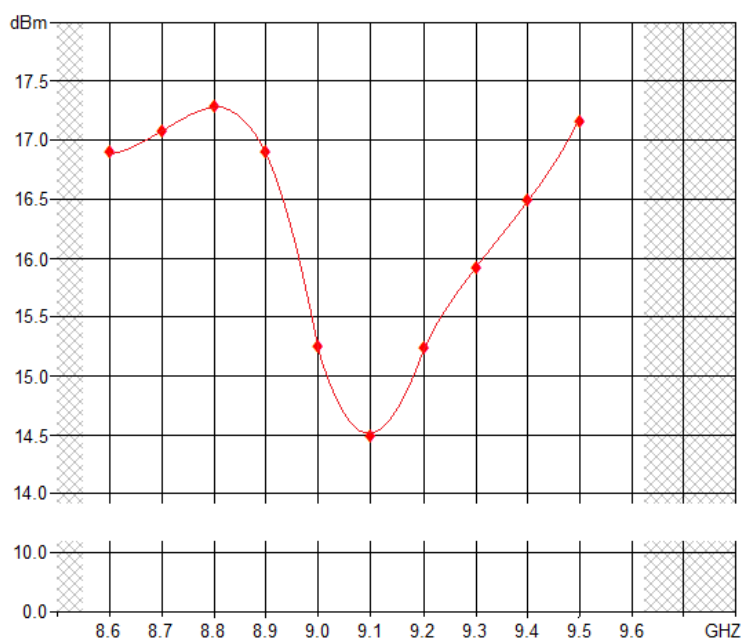

Fig. 6. Power characteristic of the Gunn oscillator depending on the frequency

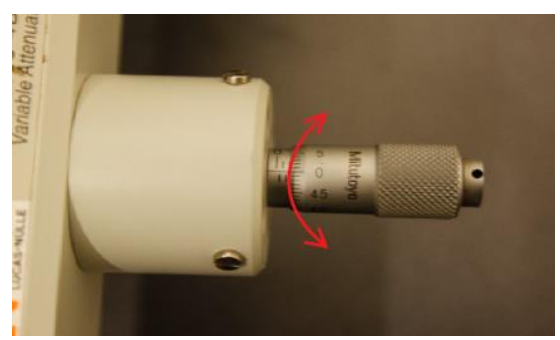

Fig. 7. The micrometric screw of the Variable Attenuator

Through these operations, the same power level of the microwave signal that meets the surface and passes through the sample (Fig. 8) to be analysed, placed inside the waveguide, at the exit of the Slotted guide segment can be ensured [9]. 

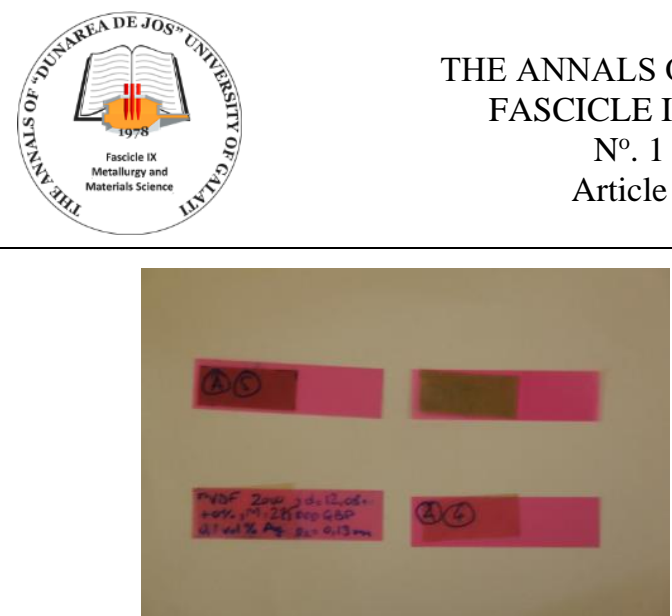

Fig. 8. The different dielectric samples

The microwave signal level before meeting the sample surface is taken by the probe placed at the beginning of the segment Slotted guide, and the microwave signal level after it crosses the sample thickness, is collected by the probe placed in the final segment of the experimental installation.

This experimental installation for the study of attenuation by absorption by a set of dielectric samples [10-12] can be used only for those samples that introduce attenuation by absorption smaller or at most equal to the value of the power of the microwave signal before meeting the sample surface.

The measurements were made on ultra-thin layers of dielectric or ferroelectric materials [13-15], obtained by the team of researchers and physicists from the Physics Faculty of the "Alexandru Ioan Cuza" University of Iasi [16].

A relevant result regarding the errors that could occur in absorption measurements, when there is no control of the emission power of the Gunn diode microwave generator, can be seen in the following two graphical representations (Fig. 9, 10):

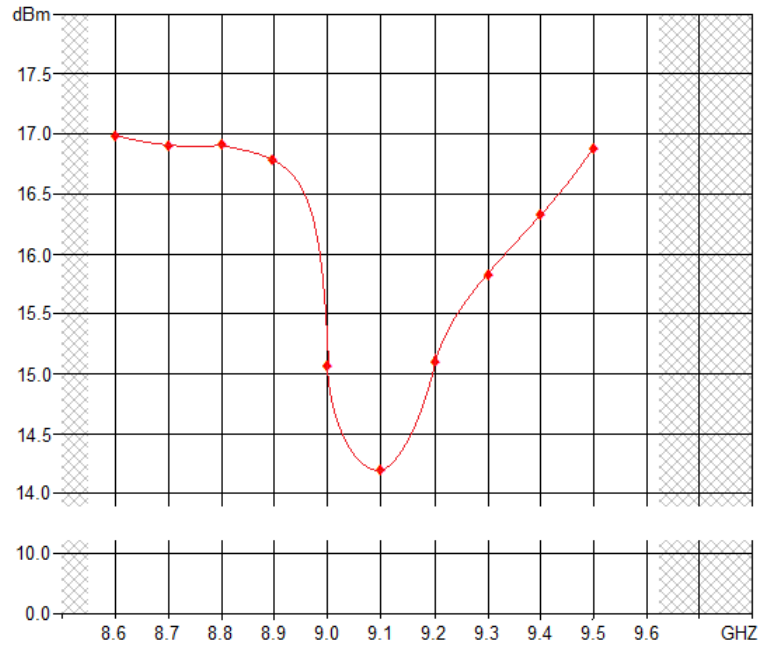

Fig. 9. Microwave power absorption feature for PVDF sample 0\% of CNT, without correction of the signal level emitted by the Gunn oscillator

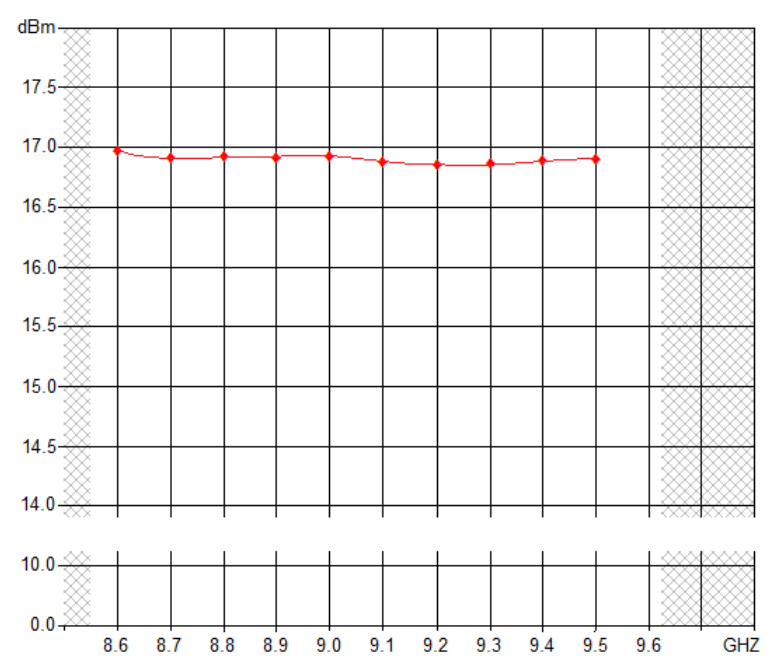

Fig. 10. Microwave power absorption feature for PVDF sample 0\% of CNT, with correction of the signal level emitted by the Gunn oscillator

Comparing the two graphical representations, the first (Fig. 9) obtained without correcting the power variations of the microwave signal emitted by the Gunn oscillator, and the second (Fig. 10) with the corrections, major differences are observed.

The experimental results, graphically represented in the first figure (Fig. 9), could lead to the conclusion that the measured sample shows an absorption at the frequency of $9.1 \mathrm{GHz}$, but in fact this decrease of the detected signal level is due to the variation of the power emitted by the Gunn oscillator, depending on the frequency [17].

\section{Conclusions}

This experimental installation is designed to study ultra-thin films of dielectric material, under the aspect of attenuating a microwave radiation located in the 8.6-9.5 GHz band, with the constant value of the electromagnetic field microwave power incident on the sample surface.

The use of samples in the form of ultra-thin layers has the advantage of being able to use microwave incident radiant powers of small values, because the level of absorption by the ultra-thin layer has a low value.

It was observed that if the ultra-thin samples show non-uniformity of concentration at the surface, then phase changes of the emergent wave occur, changes that can lead to erroneous results regarding the signal level detected by the probe.

If homogeneous samples are used and microwave signal level corrections generated by the Gunn oscillator are made, then it can be ensured an accuracy of the microwave signal level estimation, which is intended to be constant regardless of the 


\section{THE ANNALS OF "DUNAREA DE JOS” UNIVERSITY OF GALATI \\ FASCICLE IX. METALLURGY AND MATERIALS SCIENCE \\ No. 1 - 2019, ISSN 2668-4748; e-ISSN 2668-4756 \\ Article DOI: https://doi.org/10.35219/mms.2019.1.06}

frequency used, about $0.1 \mathrm{dBm}$ relative to the measured value, which ensures better accuracy than $1.5 \%$

\section{References}

[1]. ***, UniTrain-I Course - High Frequency Technology 1: "Introduction to Microwave Technology", Course no.: SO4204-9U Version 1.1.

[2]. Sharief Sami M., Ghobrial S. I., X-Band Measurements of the Dielectric Constant of Dust, Proc. Ursi Commission F 1983 Symposium. Louvain. Belgium, (ESA SP-194), June 1983.

[3]. Ghobrial S. I., Sharif S. M., Measurements of the dielectric constant of dust at $8.3 \mathrm{GHz}$, National Radio Science Meeting, Univ. of New Mexico, Albuquerque, USA, May 1982.

[4]. Postma A. J., Terlouw J. C., Calculations and measurements on an X-band microwave interferometer, Journal of Nuclear Energy. Part C, Plasma Physics, Accelerators, Thermonuclear Research, vol. 5, no. 1, 1963.

[5]. Emmanuel Saint-Christophe, René Sardos, Richard Barrue, X-band FMR measurements: establishing of relations between the complex permeability and reflection factor of ferromagnetic amorphous conductors, Journal de Physique III, EDP Sciences, 3 (10), p. 2053-2058, 1993.

[6]. Causa M. T., Passeggi M. C. G., X-band antiferromagnetic resonance measurements in KNiF3, Phys. Rev. B 32, 3229, 1 September 1985, Ashwini P. Alegaonkar, Prashant S. AlegaonkarNanocarbons: Preparation, assessments, and applications in structural engineering, spintronics, gas sensing, EMI shielding, and cloaking in X-band, Nanocarbon and its Composites. Preparation, Properties and Applications, Woodhead Publishing Series in Composites Science and Engineering, Woodhead Publishing, p. 171-285, 2019

[7]. Rakhi R. B., Preparation and properties of manipulated carbon nanotube composites and applications, Nanocarbon and its Composites. Preparation, Properties and Applications, Woodhead Publishing Series in Composites Science and Engineering, Woodhead Publishing, p. 489-520, 2019.

[8]. Raja Nor Othman, Arthur Norman Wilkinson, Carbon Nanotube Hybrids and Their Polymer Nanocomposites, Synthesis,
Technology and Applications of Carbon Nanomaterials, Micro and Nano Technologies, Elsevier Ltd., p. 29-60, 2019.

[9]. Sharief Sami M., Ghobrial S. I., X-Band Measurements of the Dielectric Constant of Dust, Proc. Ursi Commission F 1983 Symposium. Louvain. Belgium, (ESA SP-194), June 1983.

[10]. Mansor M. R., Fadzullah S. H. S. M., Masripan N. A. B., Omar G., Akop M. Z., Comparison Between Functionalized Graphene and Carbon Nanotubes: Effect of Morphology and Surface Group on Mechanical, Electrical, and Thermal Properties of Nanocomposites, Functionalized Graphene Nanocomposites and their Derivatives, Synthesis, Processing and Applications, Micro and Nano Technologies, Elsevier Ltd., p. 177-204, 2019.

[11]. Ahmet Şenocak, Erhan Demirbaş, Mahmut Durmuş, Phthalocyanine-nanocarbon materials and their composites: Preparation, properties, and applications, Nanocarbon and its Composites, Preparation, Properties and Applications, Woodhead Publishing Series in Composites Science and Engineering, Woodhead Publishing, p. 677-709, 2019.

[12]. Ghobrial S. I., Sharif S. M., Measurements of the dielectric constant of dust at $8.3 \mathrm{GHz}$, National Radio Science Meeting, Univ. of New Mexico, Albuquerque, USA, May 1982.

[13]. Emmanuel Saint-Christophe, René Sardos, Richard Barrue, X-band FMR measurements: establishing of relations between the complex permeability and reflection factor of ferromagnetic amorphous conductors, Journal de Physique III, EDP Sciences, 3 (10), p. 2053-2058, 1993.

[14]. Causa M. T., Passeggi M. C. G., X-band antiferromagnetic resonance measurements in $\mathrm{KNiF}_{3}$, Phys. Rev. B 32, 3229, 1 September 1985.

[15]. Il Sung Seo, Woo Seok Chin, D. Lee, Characterization of electromagnetic properties of polymeric composite materials with free space method, Composite Structures, vol. 66, issues 1-4, p. 533-542, October-December 2004.

[16]. Maria Teresa Buscaglia, Vincenzo Buscaglia, Massimo Viviani, Jan Petzelt, Maxim Savinov, Liliana Mitoseriu, Andrea Testino, Paolo Nanni, Catalin Harnagea, Zhe Zhao, Mats Nygren, Ferroelectric properties of dense nanocrystalline $\mathrm{BaTiO}_{3}$ ceramics, Nanotechnology, vol. 15, no. 9, p. 1113-1117, 2004.

[17]. Pozar David M., Microwave engineering, $4^{\text {th }}$ ed. John Wiley \& Sons, Inc., ISBN 978-0-470-63155-3, 2012. 